INTERNATIONAL DESIGN CONFERENCE - DESIGN 2018

https://doi.org/10.21278/idc.2018.0415

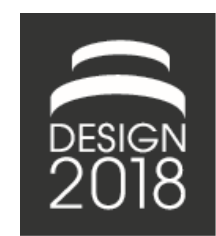

\title{
PROTOTYPING IN MECHATRONIC PRODUCT DEVELOPMENT: HOW PROTOTYPE FIDELITY LEVELS AFFECT USER DESIGN INPUT
}

\author{
L. S. Jensen, L. Nissen, N. Bilde and A. G. Özkil
}

\begin{abstract}
This paper provides a study of prototyping; with the aim of understanding how the fidelity of prototypes affects inputs by users. During development of a mechatronic padlock, 4 physical prototypes at varying fidelity were fabricated. 66 interviews with users were conducted. Users were presented with 1 of the 4 prototypes. The study finds; fidelity of prototypes affects users' feedback. Though not linearly and without unambiguity. This underlining the complexity of prototyping. A better understanding of how prototypes are perceived can help designers in establishing prototyping strategies.
\end{abstract}

Keywords: prototyping, prototyping strategies, engineering design, product design

\section{Introduction}

Prototypes are essential in product development and it is generally accepted that designers utilize prototypes in various ways and formats throughout the design process.

Historically, the costs associated with prototyping accounted for as much as $50 \%$ of the costs in product development (Cooper, 2001). In recent years we have witnessed vastly declining expenses for the tools to conduct cost effective and functional prototyping. This is substantiated by Camburn's statement: "additive manufacturing (3D printing), open-source software repositories, and reconfigurable electronic hardware enable a drastic cost reduction in single unit manufacturing" (Camburn et al., 2017). In other words; the costs and 'barrier of entry' for prototyping in product development is lowering for e.g. the development of mechatronic consumer hardware. Such opportunities introduce new questions on what defines best practice in when, how and what to prototype. Essentially, answers to such questions require designers to carefully establish prototyping strategies (Drezner, 1992; Christie et al., 2012) and plan what should be explored and learned from prototyping.

A general key objective of prototyping is to obtain sufficient knowledge on how to progress in a project with minimal expenditure in terms of time and cost (Otto and Wood, 2003). In e.g. user-centered design, prototypes are presented to relevant stakeholders to explore human factors in design. Often such prototypes do not resemble the full functionality or quality of the final product. In these situations, abstractions of prototypes can influence how the concept is communicated and stakeholders can make unintended judgements of the concept (Crilly et al., 2004). In this way, the 'quality' of prototypes influence the feedback stakeholders provide (Deininger et al., 2017). Currently, only a fragmented understanding of the fundamental question "What does prototypes prototype?" (Houde and Hill, 1997) is documented in engineering design literature.

This paper aims to contribute to the understanding of how prototype fidelity levels affect user feedback. It is our hope that insights from this study can be applied by designers when establishing prototyping 
strategies in product development projects. Therefore, this study is focused on the following research question:

- RQ1: How the fidelity of prototypes (representing a mechatronic concept) affect test users' perception of a concept?

Our findings are based on interviews with 66 test users of a mechatronic padlock. All test users were exposed to one of four different prototypes of the padlock design. The four prototypes all vary in their fidelity but represent the same product interfaces. All test users were interviewed in a semi-structured format by following an interview protocol with particular focus on the utility, usability and desirability of the mechatronic padlock. A comparison of these results leads to our conclusions on the influence which fidelities of prototypes have on the test-user feedback, which can be seen as recommendations in the establishment of prototyping strategies.

\section{Background}

A prototype is an artefact that approximates a feature (or multiple features) of a product, service, or system (Otto and Wood, 2003) and a large body of research supports the positive influences of prototypes in design activities (Wall et al., 1992; Schrage, 2010). Prototypes and prototyping is an integrated part of design activities and a range of different objectives, characteristics and purposes of prototyping is documented to take place in the various stages of product development (Jensen et al., 2016).

A current research trend is stressing the need for designers and industry practitioners to actively use prototypes as a key part of the product development process. (Menold, 2017) has proposed the 'Prototype for X framework' to support structured prototyping activities, (Hostettler et al., 2017) have proposed the 'TAF Agile Framework', where prototyping is a key activity for crystalizing knowledge fast throughout design teams, (Jensen et al., 2017) propose that organizations adapt a mindset to reflect a 'prototrialing' culture to effectively elicit unknown unknowns. A shared characteristic for this new research trend is that designers and practitioners are to establish prototyping strategies as part of the development process (Christie et al., 2012; Camburn et al., 2017; Lauff et al., 2017).

A prerequisite for establishing well-founded strategies on prototypes is that designers either possess (e.g. through experience) or have access to a knowledge base on various aspects of prototyping to provide adequate guidance for decision-making and execution of the strategic elements of prototyping. (Camburn et al., 2013) have proposed how these strategic aspects of prototyping can be described in the five overall categories: Scale, Integration, Logistics, Embodiment and Evaluation (Camburn et al., 2013).

$\$$, we only have a fragmented understanding of what defines best practice in prototyping for each of the five categories proposed by Camburn. On the overall level, we know that prototyping can be a helpful tool to reduce design complexity (Gerber, 2009) and that experienced designers rely heavily on prototypes to quickly test ideas and to generate new ones. It is also widely accepted that testing with end-users is important for the viability and usability aspects of design as it leads to early identification of any problems (Heaton, 1992; Bailey and Konstan, 2003). However, it is not unambiguous how prototyping with end-user should be conducted;

(Lim et al., 2006) argues that when using low fidelity prototyping techniques, it becomes harder to claim whether the evaluation findings originate in the actual concept of the system or in the innate characteristics of the prototype (Lim et al., 2006). In Studies by (Sauer and Sonderegger, 2009; Sonderegger and Sauer, 2010), it is documented how stakeholders' evaluation of the functionality of a design was related to the design aesthetics of a prototype (Sauer and Sonderegger, 2009; Sonderegger and Sauer, 2010). Similar findings are documented in a recent and related study by (Deininger et al., 2017). In contrast, (Reid et al., 2013) find that in some cases, product representation did not influence preferences of end-users whereas in other cases it did (Reid et al., 2013).

As expressed by (Camburn et al., 2017), there is a need for establishing "a clearer understanding of quantified information gained from a prototype" and for clarifying "the effect of fidelity on consumer emotional preference". In order to further explore such lack of clarity, we conducted this study with a focus on various prototype formats and how they were perceived by potential end-users. This differs 
from previous work by (i) Exclusively focusing on physical prototypes. (ii) Focusing on a mechatronic product testing and end-consumers' perception thereof. (iii) Utilization of recent advancements in 'prototyping tools' such as laser cutting, $3 \mathrm{~d}$ printing and reconfigurable electronic hardware for the fabrication of prototypes.

The rest of the paper is organized as follows; The following section describes our methodology and describes how the data is collected and handled. Next, we present our findings based on our analysis of the data corpus which is then followed by a discussion of the results. Finally, we conclude the paper with our reflections and provide directions for future research.

\section{Methodology}

This study was conducted as part of a product development project of a mechatronic padlock. The product is being designed by a small start-up company. It is the ambition of the start-up to sell the final padlock to institutions that offer locker room access to their users. Three examples could be educational institutions, sport facilities and libraries. During the conceptual stage of the development project designers from the start-up collected feedback from test users by utilizing four different prototypes at different stages of fidelity. The prototypes are presented in (Figure 1), and only one prototype was presented to each participant. We believe that two primary reasons make the mechatronic padlock suitable for this study. (i) The basic concept of a padlock is considered known by the general public. (ii) The code-input interface for this padlock concept is not widely domesticated and hereby suitable for a usability study. In order to collect insights and feedback, semi-structured interviews were conducted by following an interview protocol.

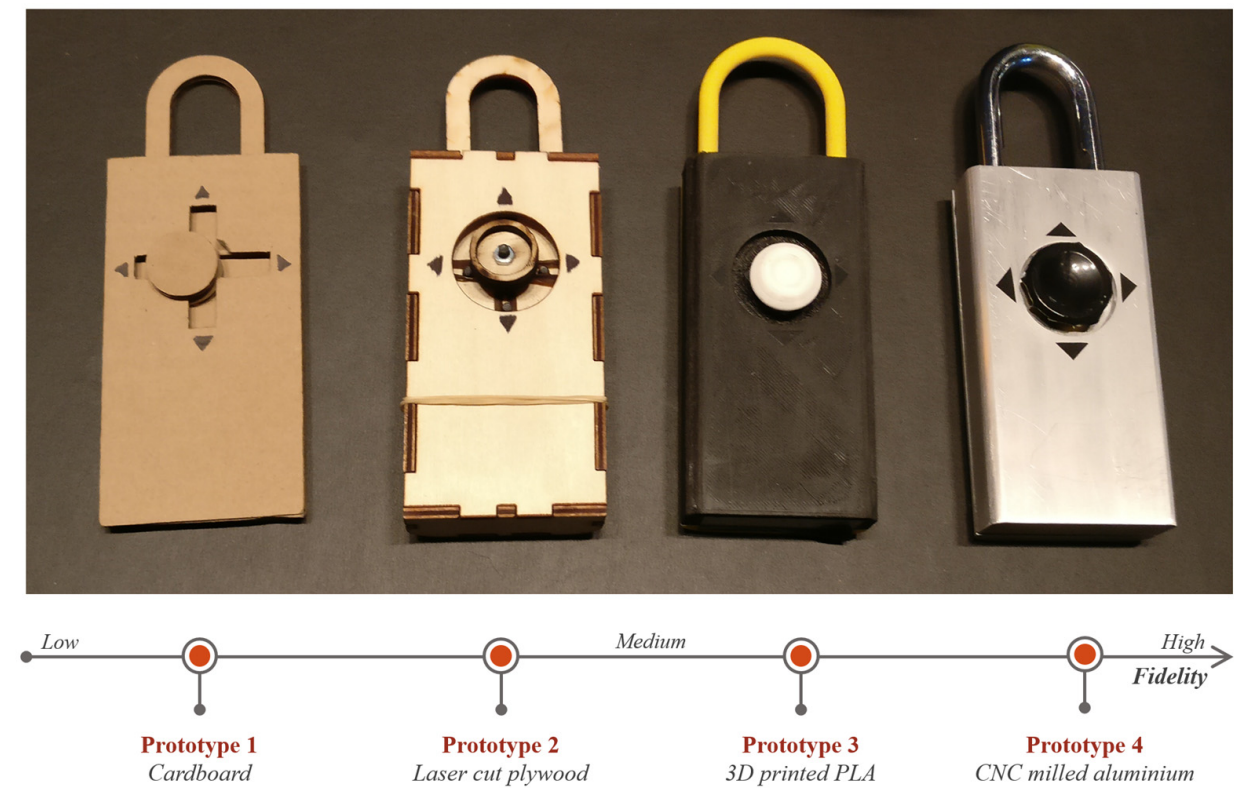

Figure 1. Photograph of the four prototypes of the padlock design used for the study; The process bar in the bottom illustrates how the fidelity of the prototypes increases from left to right

\subsection{Participants and the mechatronic padlock}

The study was conducted with a total of 66 participants heterogeneously distributed throughout different social parameters. The participants varied from 13 to 48 years of age and the division of gender was 34 males and 32 females. All participants represent the target group for the mechatronic padlock, as they are frequent users of one more of the three facilities; educational institutions, sport facilities or libraries where there currently are locker rooms available to the users. 


\subsection{Interview protocol and focus}

The interviews were conducted by an interviewer and a silent observer at the participant's respective location, e.g. at office communities and public study halls. The interview protocol used was inspired by a recent and related study by (Deininger et al., 2017): The first part of the interviews collected overall background information on the participant, the second part concerned questions to evaluate the participant's understanding of the padlock concept and the last part evaluated the perceived utility, usability and desirability by the participant.

The interview guide also consisted of three qualitative questions where participants could respond how they understood the prototype, what they liked and what they would change. Additional followup questions were encouraged by the interviewer. Whenever possible responses were coded directly and further comments were noted by the silent observer. Additionally, one question was based on the Microsoft Product Reaction cards, which is a dedicated card deck for usability testing (Benedek and Miner, 2002). The card deck is designed by usability researchers at Microsoft and the total deck consist of 118 words and phrases, which are considered to reflect positive, neutral and negative aspects of a product. The use of the reaction cards is documented to support users in telling a rich and revealing story on their experience (Barnum and Palmer, 2010). On the other hand, the use of the reaction cards can also introduce a bias, which should be considered as a reservation for the result of the study.

The participant was presented with a selection of 19 words, with the option to select 3 of these words.

\subsection{Prototypes}

When prototypes of different fidelity levels are compared, a measure of applicable comparison is convenient. In a case study by (Zhang et al., 2012), concept interfaces are resembled across all prototypes for comparison. Further, only some dimensions of the prototypes are varied. A similar approach is adapted for this study by maintaining the padlock concept interfaces with a directional code input on the front. Variance was only introduced in dimensions such as in materials, ergonomics, look and feel of the prototypes. All prototypes were designed and fabricated by the designers of the padlock concept with the exception of prototype 4, where the aluminum housing was CNC milled with the support of a workshop technician (Table 1).

Table 1. Overview of time, estimated costs, tools and resources needed for the fabrication of the four prototypes

\begin{tabular}{|c|c|c|c|c|}
\hline & Prototype 1 & Prototype 2 & Prototype 3 & Prototype 4 \\
\hline $\begin{array}{l}\text { Time: Design \& } \\
\text { construction }\end{array}$ & 30 minutes & 2 hours & 4 hours & 20 hours \\
\hline $\begin{array}{c}\text { Fabrication: } \\
\text { Estimated cost }\end{array}$ & $1 €$ & $20 €$ & $50 €$ & $400 €$ \\
\hline $\begin{array}{c}\text { Tools \& } \\
\text { Resources }\end{array}$ & $\begin{array}{l}\text { Cardboard } \\
\text { Scissor } \\
\text { Knife } \\
\text { Glue }\end{array}$ & $\begin{array}{c}\text { Plywood } \\
2 \text { X Rubber band } \\
\text { Bolt \& nut } \\
\text { CAD layout } \\
\text { Laser cutter }\end{array}$ & $\begin{array}{c}\text { ABS filament } \\
\text { Button mechanism } \\
\text { Glue } \\
\text { CAD layout } \\
\text { 3D printer }\end{array}$ & $\begin{array}{c}\text { Shackle } \\
\text { Nylon Button } \\
\text { Input mechanism } \\
\text { PCB } \\
\text { Battery } \\
\text { Aluminum } \\
\text { CAD layout } \\
3 \text { axis CNC } \\
\text { Machine technician }\end{array}$ \\
\hline
\end{tabular}

\subsection{Data analysis}

The data generated from the interviews was a combination of quantified information (answers on a Likert scale and choice of reaction cards) and qualitative information recorded by the silent observer. The data was transcribed and analyzed by two researchers with advanced degrees in engineering design and the designers from the start-up company. The quantified and directly coded data from the interviews 
were synthesized and visualized as presented in Figure 3 - Figure 7. The authors worked collaboratively on the coding and analysis of the qualitative aspects of the interviews in order to further comprehend the reasoning provided by the participants. As this activity were carried out as a concurrent and collaborative activity it was not possible to verify this coding through an interrater reliability evaluation. The results of such activity is presented in Figure 2.

\section{Results}

\subsection{Utility}

This first part of the interviews had the purpose of evaluating if participants understood the padlock interface concept. The comprehensions thereof are summarized in Figure 2, and they were based on the participants' ability to answer the question: "Can you explain how you would unlock the prototype?" Prototype 1 scored the highest number of correct answers together with prototype 3 , while prototype 2 and the 'high-fidelity' prototype 4 received the majority of incorrect answers. From the coded feedback from the participants, it is indicated that the slits in the cardboard of prototype 1 and the simplistic layout of prototype 3 helped the participants understand the intended use of the padlock. In interviews involving prototype 2 , a group of participants $(n=3)$ perceived that the joystick could rotate. This can be exemplified by participant 57's statement: "I would turn this wheel a bit to the left, and then a little more to the right, and then back again to hit the correct numbers" - even though no numbers were present on the prototype.

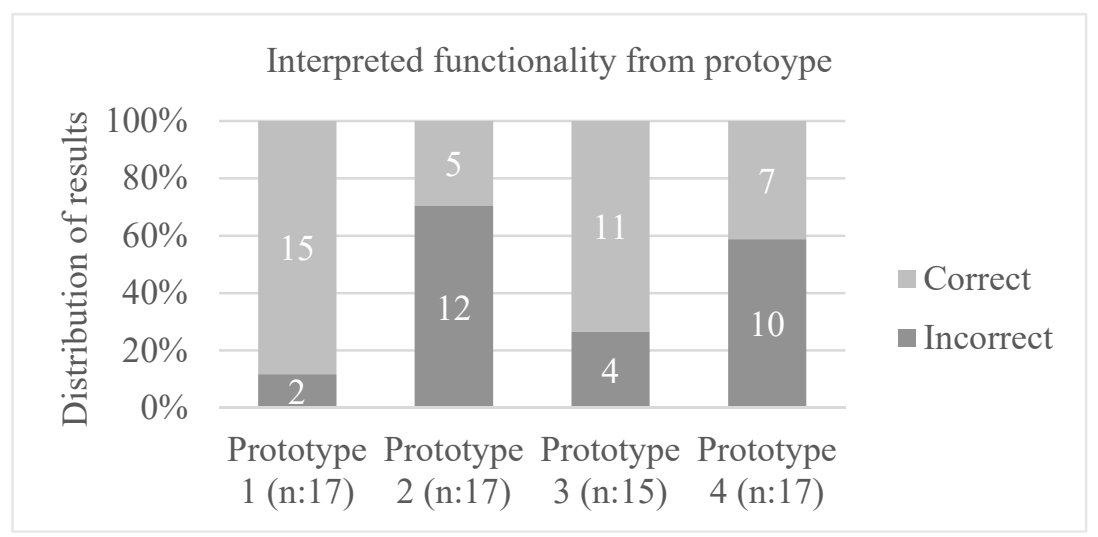

Figure 2. Evaluation of the four prototypes in terms of their ability to communicate the working principle of the padlock concept's locking mechanism

The incorrect answers on prototype 4 were by a group of participants $(n=2)$ based on technological expectations. Participant 42 was expecting an app to unlock it, and participant 32 was assuming there would be a display with numbers on the product.

A frequent and equally often represented concern across all prototypes were the participants' concern about their ability to remember a directional code if they were to use it at a locker. In order for the participants to express their opinion on the prototypes, they were asked how they would evaluate the interface of the locking mechanism to work in practice. The answers were recorded on a Likert scale and are summarized in Figure 3.

Figure 3 presents how prototypes 1 and 2 receive evaluations with a lower distribution than prototypes 3 and 4. It was found that the prototype 4 in high fidelity received more concrete feedback, e.g. exemplified by Participant 39: "In a practical scenario dirt and gravel could penetrate the lock at the button." Though the coding it was concluded that examples of concrete feedback was given in 8 cases for prototype 4 . Prototype 3 had the second highest level of concrete feedback with 5 cases. A hypothesis for the more concrete feedback on higher fidelity prototypes and average evaluation of the low fidelity prototypes can be the higher level of abstraction needed to interpret low fidelity prototypes. 


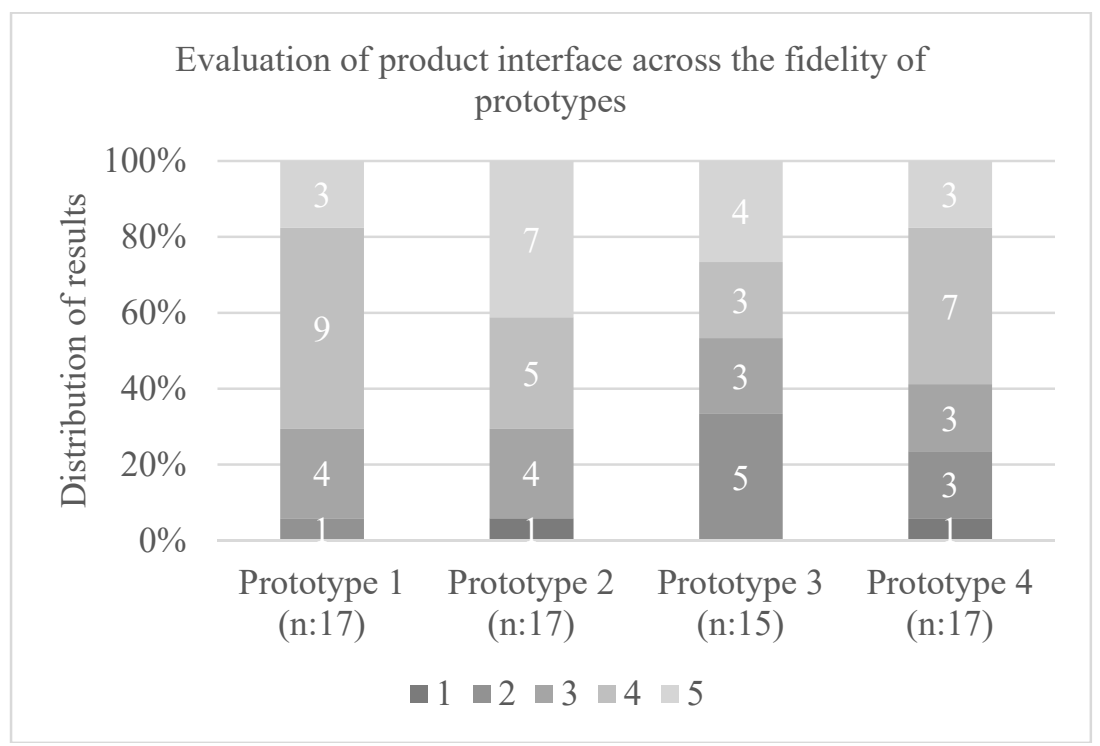

Figure 3. Overview of the participants' evaluation of the locking mechanism of the different prototypes, where 1 is very unattractive and 5 is very attractive

\subsection{Usability}

The second section of the results describes the participants' perceived usability of the four prototypes. Questions prompted the participants to provide their view on the usability of the padlock concept by assessing the interface of the prototype. The participants were asked about how 'easy' it was to understand the interface and how to interact with it. The participants were then asked to what extend they found the concept to be a user-friendly solution [to themselves]. The results are summarized in Figure 4.

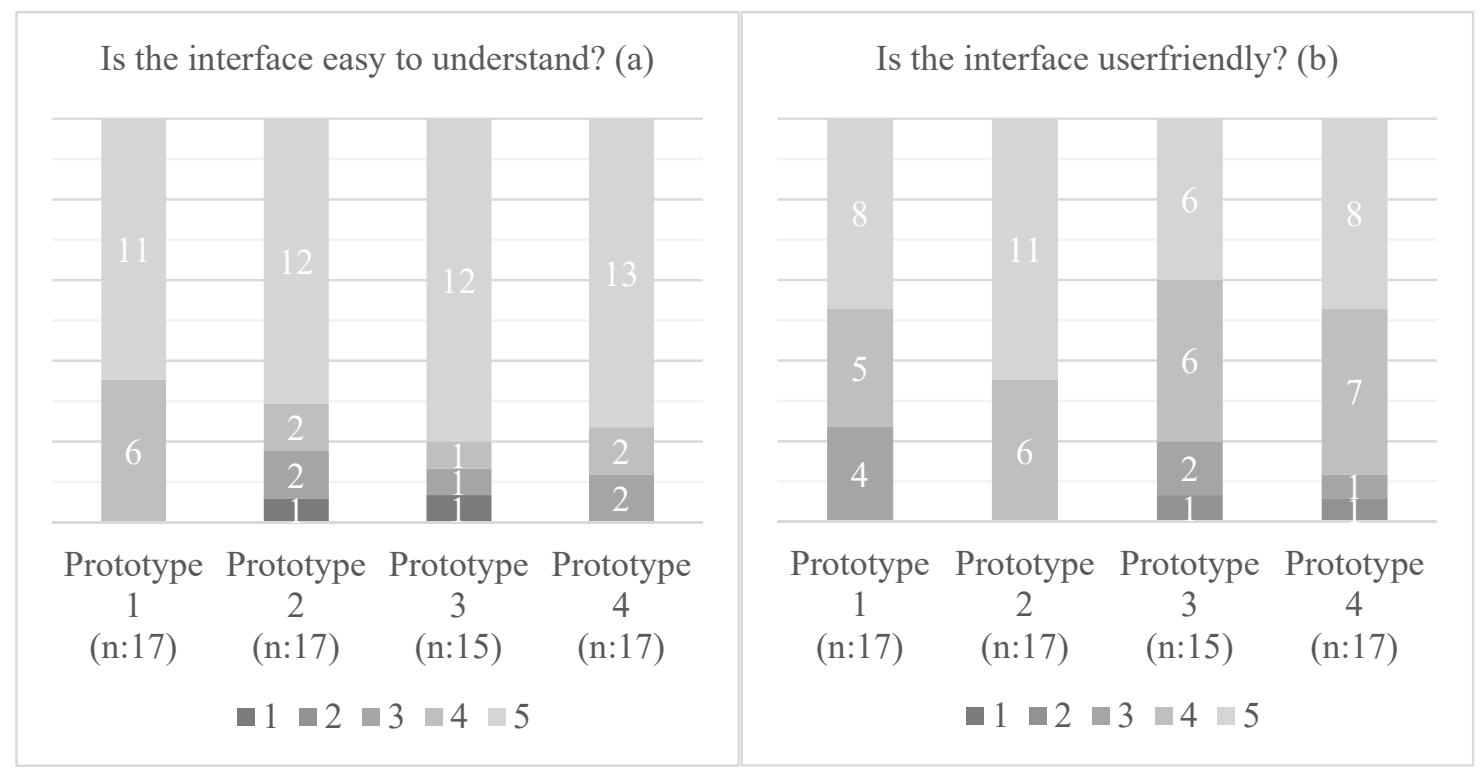

Figure 4. Overview of the participants' view on the ease of understanding of the prototype interface (a, left) and to what extend they found the concept user friendly (b, right). In both cases 1 is very low and 5 is very high

We observe that there is only a relatively low variety in how the participants evaluate the ease of understanding the interface of the prototypes (Figure 4a). The user-friendliness of the interfaces (Figure $4 \mathrm{~b})$ shows a more mixed evaluation of the prototypes. In terms of mean values, prototype 2 receives the 
best evaluation followed by prototype 4 . The prototypes of higher fidelity, prototypes 3 and 4 , are evaluated with larger distribution in the results relative to prototypes 1 and 2.

\subsection{Desirability}

The objective of the final part of the study is to allow the participants to articulate their reflections and experience with the prototypes. By doing so they were encouraged to emphasize which parameters of the prototype they were most fond of and where they could detect room for improvements or changes. Figure 5 summarizes words chosen by the participants from the Product Reaction Cards introduced in the methodology. Figure 6 represents to what extend the participants found the concept attractive, and Figure 7 presents the participants' interests in improving the concept through changes.

When asked to select three reaction cards to describe the prototype, 'Easy to Use' and 'Simplistic' were the most frequent words chosen by the participants. Words like 'Fun', 'User-friendly' and 'Unsafe' became less frequent with the increased level of fidelity. For instance, Participant 5 used the following phrases to describe prototype 2: "It doesn't look secure. The shackle could easily be cut open." This concern of safety was further referred to by three other participants. and participant 31 described prototype 4 using this phrase: "It's very robust", which was mentioned by additional three participants. This indicates that some participants cannot be expected to abstract from what the prototype 'is' and what it is supposed to resemble.

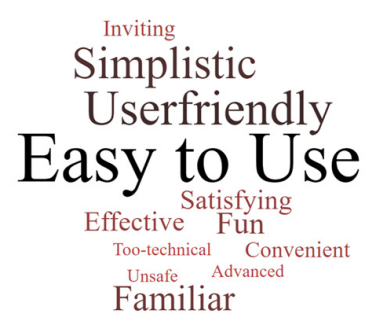

Prototype 1

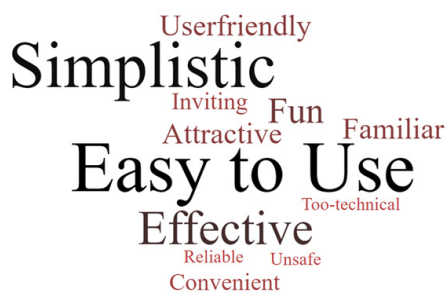

Prototype 3

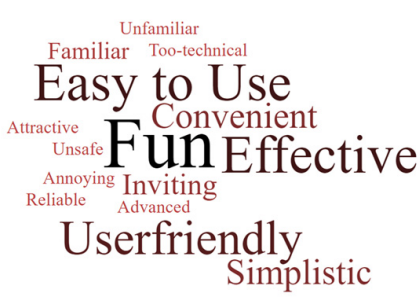

Prototype 2

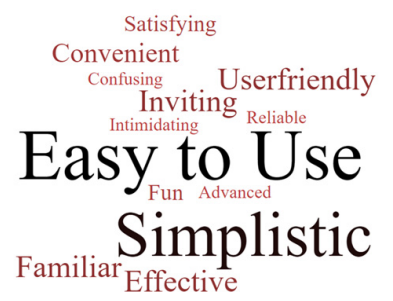

Prototype 4

Figure 5. Word clouds based on the participants' choice of reaction cards to best describe their point of view on the concept; Each cloud represents each of the four prototypes

Figure 6 presents the results of the study determining to which extent to the participants found the concept attractive. The quantitative results do not articulate any strong variations in how the prototypes were evaluated. The qualitative data, however, gives a more detailed view on the participants' focus and thoughts. A range of examples are how; For prototype 1, participant 1 expressed: "Easy solution to a known problem". For prototype 2, participant 18 expressed "I like the combination pattern, it is fun and innovative." For prototype 3, participant 14 expressed fondness of the size and added "I like the feeling of the joystick". And finally for prototype 4, participant 32 said "It looks like what it is. It is not fancy". 


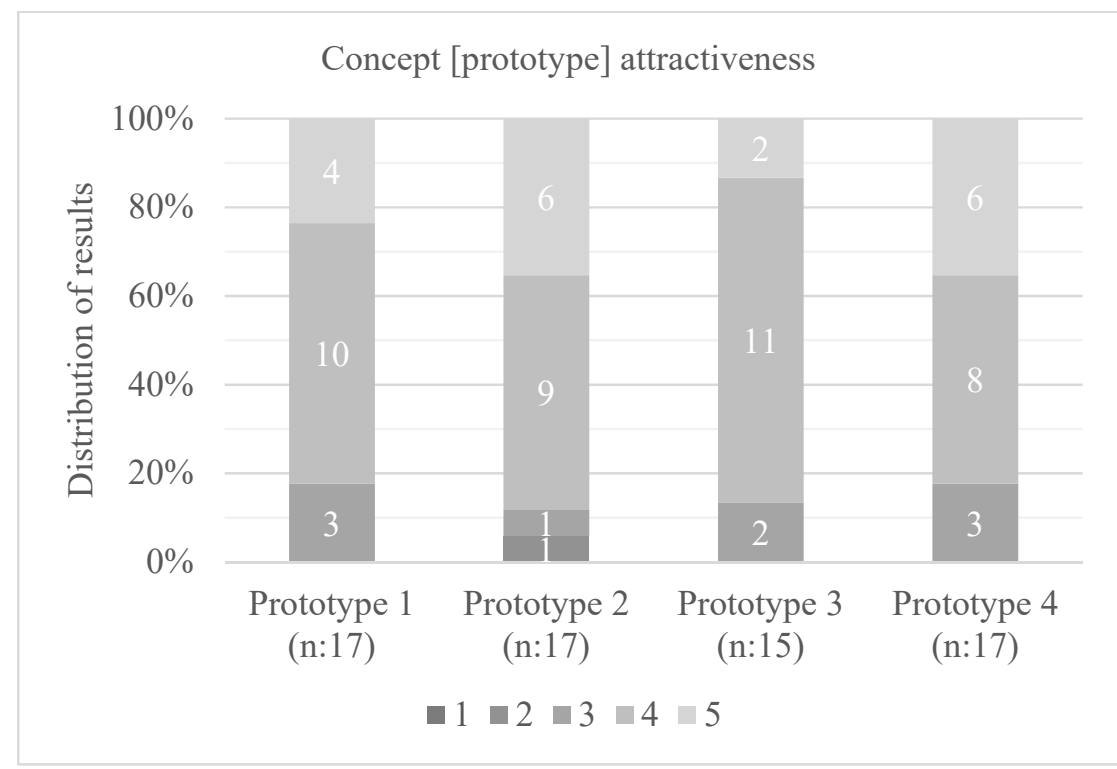

\section{Figure 6. Evaluation of the extent to which the participants found the concept attractive where 1 is very little and 5 is very much}

Figure 7 presents the participants' interests in performing changes to the padlock concept. As seen in Figure 7 the participants were less interested in suggesting changes to prototypes 1 and 4, whereas they were more interested in changes to the 'mixed-fidelity' prototypes 2 and 3. Concerning prototype 1, Participant 23 claimed: "I don't know what to change based on this cardboard model".

However, some participants did suggest changes to prototype 1. A group of 6 Participants all wanted to change aspects related to the length of the code or the way it was 'put in'. Prototypes 2, 3 and 4 all received inquiries about changing the size of the prototypes (in total $n=11$ ). For prototype 2, participant 29 expressed concern of safety: "I don't really know if it will be safe, this [the shackle, red.] is pretty small compared to the rest". For prototype 3, 3 participants addressed size and feeling of joystick. Finally, in prototype 4, 4 participants addressed the looks of the prototype interface; how colors could be better in remembering code or how it would be more appealing to themselves.

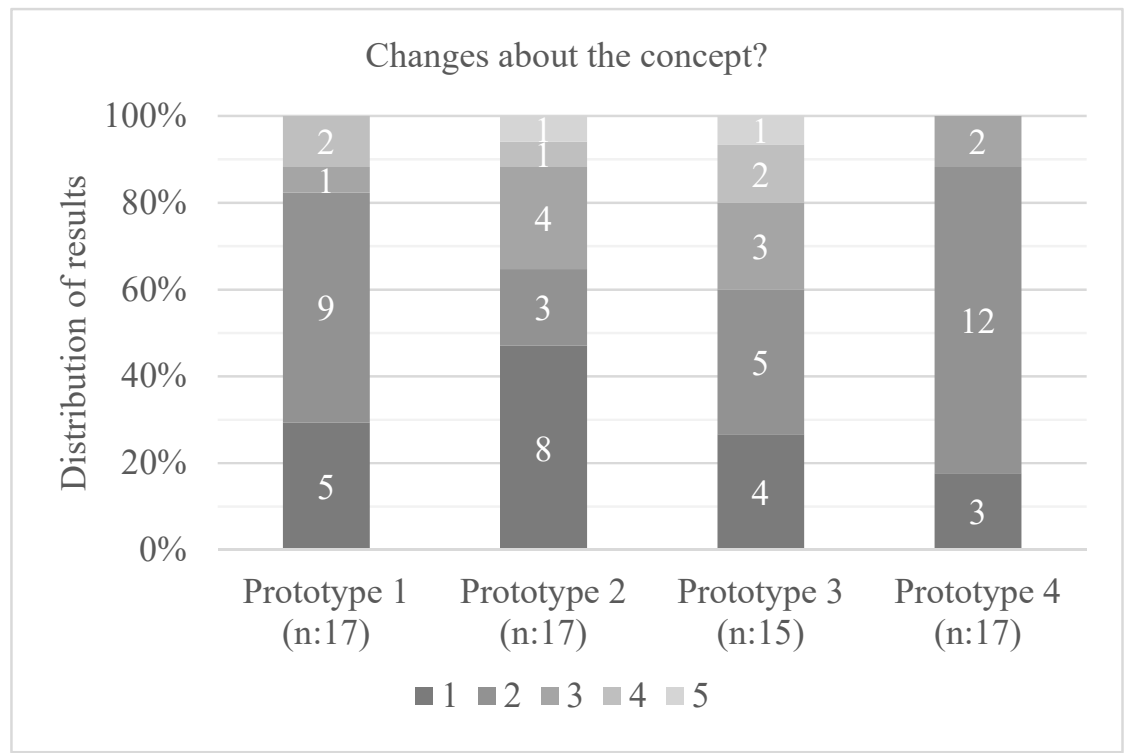

Figure 7. Evaluation of the participants' interests in improving the concept through changes, where 1 is very little and 5 is very much 


\section{Discussion}

In this section we evaluate the results of the study, and what conclusions can be made in relation to the research questions of the study. Last, we present perspectives on how practitioners can apply these results when establishing prototyping strategies.

\subsection{Utility}

The results document rather large variations in the test-users' ability to interpret the working principle of the mechatronic padlock concept. According to the results, prototypes 1 and 3 are better at communicating the working principle to test-users, whereas an overarching number of test-users misinterpreted prototypes 2 and 4 . When evaluating the qualitative data, two characteristics were misinterpreted by the test-users. On prototype 2, the joystick button was (unintentionally) able to rotate, which made a group of 3 participants recognize it as an intended functionality. The authors evaluate that the high fidelity of prototype 4 created unintended noise for determining the working principle. The ergonomic shape, a functional shackle and LED backlights drew attention towards other aspects of the concept rather than functional interface; only for prototype 4 it was suggested by 2 participants that a digital (app or interface) solution was the intended interface for the unlocking mechanism.

For the broader evaluation of how the participants perceived the interface to perform in practice, we observe how the highest mean score was devoted to prototype 2 - this despite the previously highlighted unintended rotating functionality of the joystick. The evaluations do however show a gradual increase in the distribution of evaluations. From the qualitative feedback, we conclude that the increased fidelity helped participants to imagine use cases and associated potential benefits and challenges.

\subsection{Usability}

Only a relatively low variety was recorded in the participants' evaluation of the ease of understanding the interface. For the usability, we observe again an increasing distribution in the results emerging along with the increasing fidelity of the prototypes. In the qualitative feedback, a pattern emerged; participants interacting with prototypes 1 and 2 primarily expressed opinions on the underlying concept and its functionality. These opinions were expressed in combination with questions for clarification in the working principle. Participants interacting with prototypes 3 and 4 articulated their satisfaction with or skepticism on the interaction functionality, how it felt, and other physical attributes like size and color. When collecting qualitative feedback, the interviewers observed how the participants were less likely to interact with prototypes 1 and 2 compared to prototypes 3 and 4 of higher fidelities.

\subsection{Desirability}

The word clouds presented in Figure 5 only show slight variations in terms of occurrences, which indicate a certain degree of commonality in the perception of the prototypes and the padlock concept. The two words 'Fun' and 'Unsafe' occurred less frequently along with increase in prototype fidelity. This underlines the statement that some participants are biased by the particular fidelity represented by the prototype and need a certain level of fidelity to consider the concept a 'safe'.

When evaluating the participants' view on the attractiveness of the concept, all four prototypes were evaluated with only slight variation. Variations in the participants' points of view were to a higher extent articulated when encouraged to provide qualitative feedback. For nine participants the feedback for prototype 1 was related to the underlying the interaction concept for the padlock, while feedback for prototypes 2 six participants articulated the joystick. Feedback for Prototype 3 were in ten cases focused on the joystick and in eight cases its interface. This was so both in terms of the functionality $(n=6)$ and more detailed aspects like the feel of it. Feedback for prototype 4 were similar to that of prototypes 2 and 3, but in a less pronounced way. This trend is further emphasized and mirrored in the participants' interest in improving the concept through changes. We believe that this relates to the fidelity of the prototypes: For prototype 1, the fidelity is so low that it hinders the participants' perception and ability to evaluate the concept. Contrary to this, the fidelity of prototype 4 is very high and the participants are focused on the finish and the details, and are reluctant to provide critique. The 'mixed fidelity' of 
prototypes 2 and 3 provide a perception of concrete but not finalized design which invites for changes and improvements.

\subsection{What does this study tell?}

With this study, we set out to investigate if the fidelity of prototypes affects users' perception of the concepts that are being tested. We conclude that prototype fidelity does have an influence on how the concept is perceived by test users. However, the results are not linear and without unambiguity. This underlines the previous finding: Prototyping is a complex activity and no one prototype is equally suitable for all design questions (Deininger et al., 2017).

We find that when evaluating how the utility of a concept is perceived, too many functions incorporated in the prototype can introduce noise which may remove focus from the particular aspect of the product design that the designer seeks feedback on. Also unintended functionality of a prototype can result in incorrect interpretations of concept functionality.

In terms of usability, our results suggest that low fidelity prototypes are (resource) effective tools in understanding the needs of test users. Buur and Andreasen have expressed that prototyping is a way of buying information about a future product or service (Buur and Andreasen, 1989). As presented in Table 1 there is a 1:400 cost ratio and a 1:20 ratio in fabrication time between Prototype 1 made of cardboard and Prototype 4 of $\mathrm{CNC}$ milled aluminum. When balancing the resources spend with the design insights obtained it is underlined that low fidelity prototypes can be of value and allow for a larger number of design iterations within the same budget constraints.

The findings also indicate that for more concrete evaluations of design solutions, prototypes of higher fidelity can be more suitable. This as participants can more clearly articulate their points of view on particular design aspects that are part of the prototype embodiment. Further, not all stakeholders can be expected to have the level of abstraction necessary to provide rich feedback on a low fidelity prototype. Based on our findings we recommend practitioners to carefully plan their prototyping activities. Part of this process can include; (i) The design questions will influence what design medium can be utilized for a prototyping activity. When low fidelity prototypes can be used we recommend a large number of low fidelity prototype iterations over few high fidelity iterations. (ii) To conduct fast pre-studies to verify that the exposed prototypes are perceived as intended. (iii) To consider the inclusion of more than one type of prototype to obtain more varied feedback. (iv)To collect a combination of qualitative and quantitative data for varied results.

\section{Conclusion}

This paper presents a study on how the fidelity of prototypes affects the feedback provided by test users. Through 66 semi-structured interviews, participants were presented to one of four different physical prototypes of varying fidelity. The interviews focused on feedback provided by the participants within the three topics: Utility, usability and desirability. Based on the results thereof, we conclude that the fidelity of prototypes affects the feedback from test users - however not in a linear way and not without ambiguity, which underlines the complexity of prototyping activities.

We encourage designers and practitioners to consider the results and recommendations of this and related studies when establishing prototyping strategies. Further, we encourage future research in determining the effect of various design activities on the fidelity of prototypes. This study concerned a concept's desirability to end-users. Further studies could concern how fidelity of prototypes affects assessments of a concepts viability and feasibility by relevant stakeholders.

Several limitations of this study could be addressed in future work; it has only been possible to present a subset of the results from this study in this work. Future work could expand the number of test users and differentiate their demography and cultural backgrounds. Moreover, it could be supported by more in depth analysis and cross evaluation of the results.

\section{Acknowledgements}

The authors would like to thank Martin Meister, Workshop Manager at DTU Skylab, for his support in the fabrication of the prototypes used in this study. 


\section{References}

Bailey, B.P. and Konstan, J.A. (2003), “Are informal tools better?: comparing DEMAIS, pencil and paper, and authorware for early multimedia design”, Proceedings of the SIGCHI Conference on Human Factors in Computing Systems (CHI '03), Ft. Lauderdale, USA, April 5-10, 2003, ACM, New York, pp. 313-320. https://doi.org/10.1145/642611.642666

Barnum, C.M. and Palmer, L.A. (2010), "More than a feeling: understanding the desirability factor in user experience", CHI'10 Extended Abstracts on Human Factors in Computing Systems (CHI EA '10), Atlanta, USA, April 10-15, 2010, ACM, New York, pp. 4703-4716. https://doi.org/10.1145/1753846.1754217

Benedek, J. and Miner, T. (2002), "Measuring Desirability: New methods for evaluating desirability in a usability lab setting", Proceedings of Usability Professionals' Association Conference / the 11th Annual UPA Conference, Orlando, USA, July 8-12, 2002, pp. 8-12.

Buur, J. and Andreasen, M.M. (1989), "Design models in mechatronic product development", Design Studies, Vol. 10 No. 3, pp. 155-162. https://doi.org/10.1016/0142-694x(89)90033-1

Camburn, B., Viswanathan, V., Linsey, J., Anderson, D., Jensen, D. et al. (2017), "Design prototyping methods: state of the art in strategies, techniques, and guidelines", Design Science, Vol. 3, pp. E13. https://doi.org/10.1017/dsj.2017.10

Camburn, B.A., Dunlap, B.U., Kuhr, R., Viswanathan, V.K., Linsey, J.S. et al. (2013), "Methods for Prototyping Strategies in Conceptual Phases of Design: Framework and Experimental Assessment", Proceedings of the ASME 2013 International Design Engineering Technical Conferences and Computers and Information in Engineering Conference, Vol. 5, Portland, Oregon, USA, August 4-7, 2013, ASME, pp. V005T06A033. https://doi.org/10.1115/DETC2013-13072

Christie, E.J., Jensen, D.D., Buckley, R.T., Menefee, D.A., Ziegler, K.K. et al. (2012), "Prototyping Strategies: Literature Review and Identification of Critical Variables", Proceedings of 2012 ASEE Annual Conference and Exposition, San Antonio, Texas, USA, June 10-13, 2012, American Society for Engineering Education.

Cooper, R.G. (2001), Winning at New Products, Accelerating the Process from Idea to Launch, Basic Books, Perseus Publishing, Cambridge, Massachusetts.

Crilly, N., Moultrie, J. and Clarkson, P.J. (2004), "Seeing things: consumer response to the visual domain in product design", Design Studies, Vol. 25 No. 6, pp. 547-577. https://doi.org/10.1016/j.destud.2004.03.001

Deininger, M., Daly, S.R., Sienko, K.H., Lee, J.C., Obed, S. and Effah Kaufmann, E. (2017), "Does prototype format influence stakeholder design input?", Proceedings of the 21st International Conference on Engineering Design (ICED '17), Vol 4: Design Methods and Tools, Vancouver, Canada, August 21-25, 2017, The Design Society, Glasgow, pp. 553-562.

Drezner, J.A. (1992), The Nature and Role of Prototyping in Weapon System Development, RAND, Santa Monica CA, USA.

Gerber, E. (2009), "Prototyping: facing uncertainty through small wins", Proceedings of the 17th International Conference on Engineering Design (ICED '09), Vol. 9: Human Behavior in Design, Palo Alto, USA, August 24-27, 2009, pp. 333-342.

Heaton, N. (1992), "What's wrong with the user interface: how rapid prototyping can help", Proceedings of IEE Colloquium on Software Prototyping and Evolutionary Development, London, UK, November 11, 1992, IET.

Hostettler, R., Böhmer, A.I., Lindemann, U. and Knoll, A. (2017), "TAF agile framework reducing uncertainty within minimum time and resources", Proceedings of the 2017 International Conference on Engineering, Technology and Innovation (ICE/ITMC), Funchal, Portugal, June 27-29, 2017, IEEE, pp. 767-775. https://doi.org/10.1109/ICE.2017.8279962

Houde, S. and Hill, C. (1997), "What do prototypes prototype”, In: Helander, M.G. Laundauer, T.K. and Prabhu, P.V. (Eds.), Handbook of Human-Computer Interaction, 2nd ed., North Holland, pp. 367-381. https://doi.org/10.1016/B978-044481862-1.50082-0

Jensen, L.S., Öense, A.G. and Mortensen, N.H. (2016), "Prototypes in engineering design: Definitions and strategies", Proceedings of the DESIGN 2016 / 14th International Design Conference, Dubrovnik, Croatia, May 16-19, 2016, The Design Society, Glasgow, pp. 820-830.

Jensen, M.B., Elverum, C.W. and Steinert, M. (2017), "Eliciting unknown unknowns with prototypes: Introducing prototrials and prototrial-driven cultures", Design Studies, Vol. 49, pp. 1-31. https://doi.org/10.1016/j.destud.2016.12.002

Lauff, C., Kotys-Schwartz, D. and Rentschler, M.E. (2017), "What is a Prototype?: Emergent Roles of Prototypes From Empirical Work in Three Diverse Companies", Proceedings of the ASME 2017 International Design Engineering Technical Conferences and Computers and Information in Engineering Conference, Vol. 7, Cleveland, Ohio, USA, August 6-9, 2017, ASME, pp. V007T06A033. https://doi.org/10.1115/DETC201767173 
Lim, Y., Pangam, A., Periyasami, S. and Aneja, S. (2006), "Comparative analysis of high-and low-fidelity prototypes for more valid usability evaluations of mobile devices", Proceedings of the 4th Nordic Conference on Human-Computer Interaction: Changing Roles (NordiCHI '06), Oslo, Norway, October 14-18, 2006, ACM, New York, pp. 291-300. https://doi.org/10.1145/1182475.1182506

Menold, J.D. (2017), Prototype for X (PFX): A Prototyping Framework to Support Product Design, PhD thesis, The Pennsylvania State University.

Otto, K.N. and Wood, K.L. (2003), Product design: techniques in reverse engineering and new product development, Prentice-Hall.

Reid, T.N., MacDonald, E.F. and Du, P. (2013), "Impact of product design representation on customer judgment", Journal of Mechanical Design, Vol. 135 No. 9, pp. 91008. https://doi.org/10.1115/1.4024724

Sauer, J. and Sonderegger, A. (2009), "The influence of prototype fidelity and aesthetics of design in usability tests: Effects on user behaviour, subjective evaluation and emotion”, Applied Ergonomics, Vol. 40 No. 4, pp. 670-677. https://doi.org/10.1016/j.apergo.2008.06.006

Schrage, M. (2010), “The Culture(s) of PROTOTYPING”, Design Management Review, Vol. 4 No. 1, pp. $55-65$. https://doi.org/10.1111/j.1948-7169.1993.tb00128.x

Sonderegger, A. and Sauer, J. (2010), "The influence of design aesthetics in usability testing: Effects on user performance and perceived usability", Applied Ergonomics, Vol. 41 No. 3, pp. 403-410. https://doi.org/10.1016/j.apergo.2009.09.002

Wall, M.B., Ulrich, K.T. and Flowers, W.C. (1992), "Evaluating prototyping technologies for product design", Research in Engineering Design, Vol. 3 No. 3, pp. 163-177. https://doi.org/10.1007/BF01580518

Zhang, T., Rau, P.-L.P., Salvendy, G. and Zhou, J. (2012), "Comparing Low and High-Fidelity Prototypes in Mobile Phone Evaluation”, International Journal of Technology Diffusion, Vol. 3 No. 4, pp. 1-19. https://doi.org/10.4018/jtd.2012100101

Lasse Skovgaard Jensen, PhD student

Technical University of Denmark

Strandboulevarden 1411 tv., 2100 København Ø, Denmark

Email: 1sje@dtu.dk 